\title{
Corela
}

Cognition, représentation, langage

9-2 | 2011

Vol. $9, \mathrm{n}^{\circ} 2$

\section{Linguistique Cognitive et modèles catégoriaux : Quelques considérations épistémologiques}

Jean-Baptiste Guignard

\section{CpenEdition}

\section{Journals}

Édition électronique

URL : http://journals.openedition.org/corela/2127

DOI : $10.4000 /$ corela. 2127

ISSN : $1638-573 \mathrm{X}$

Éditeur

Cercle linguistique du Centre et de I'Ouest - CerLICO

Référence électronique

Jean-Baptiste Guignard, «Linguistique Cognitive et modèles catégoriaux : Quelques considérations épistémologiques », Corela [En ligne], 9-2 | 2011, mis en ligne le 17 février 2014, consulté le 01 mai 2019. URL : http://journals.openedition.org/corela/2127 ; DOI : 10.4000/corela.2127

Ce document a été généré automatiquement le 1 mai 2019.

\section{(c) (i) (2)(2)}

Corela - cognition, représentation, langage est mis à disposition selon les termes de la licence Creative Commons Attribution - Pas d'Utilisation Commerciale - Partage dans les Mêmes Conditions 4.0 International. 


\title{
Linguistique Cognitive et modèles catégoriaux : Quelques considérations épistémologiques
}

\author{
Jean-Baptiste Guignard
}

1 Bien que la sémantique du prototype ait toujours le « vent en poupe $»^{1}$, tout ne peut être expliqué en termes d'extensions catégorielles: il est des évidences, comme les classes dynamiquement constituées, que les modèles prototypiques standard et étendu ne peuvent expliquer. Leur objectif (cf. infra) est chaque fois de rendre compte de la multiplicité des acceptions catégorisées et fixées dans l'usage (les sens du mot), plutôt que de montrer en quoi ces acceptions peuvent faire l'objet, non seulement d'une appartenance pluri-catégorielle (cela, la sémantique étendue du prototype le montre parfois), mais en quoi les acceptions d'un même signifiant sont contextuellement dépendantes et possèdent chacune leur histoire constitutive ${ }^{2}$. Rosh elle-même (1999, p. 66), quoique plusieurs années après que son texte fondateur a forgé les outils de la sémantique du prototype, affirme la flexibilité des prototypes et leur extrême relativité contextuelle ${ }^{3}$. Tel est aussi le cas dans certains courants français d'obédience cognitive. Ainsi le lexique, pour Rastier, est-il un ensemble de « doxas figés », et la tâche du linguiste la quête des forces qui forment (et déforment) les classes lexicales et les formes sémantiques (Rastier, 2001, p. 157). Face, figure, visage dans la classe (ou taxème) des dénominations de visage, connaissent des constantes historiques relatives aux contextes d'emploi (religieux, et populaire en particulier) et dépendantes de traits de valorisation (mélioratifs pour face au XIIème siècle par ex.). Comparativement, la Linguistique Cognitive (LC), dont le modèle langackérien reste le plus abouti, recourt à la sémantique du prototype pour décrire la complexité des catégories (y compris grammaticales), leurs écarts et recoupements, comme pour décrire les nœuds sémantiques et les attributs qui permettent les rattachements des morphèmes lexicaux à une ou plusieurs catégories. Or, dans chaque cas de figure l'objet diffère considérablement, car le rapport de la constitution et de l'organisation interne d'une catégorie à la polysémie lexicale est très incertain ${ }^{4}$. Une telle approche ne saurait être épistémologiquement acceptable qu'à considérer, comme il semble que ce soit le cas en $\mathrm{LC}^{5}$, que la catégorisation lexicale 
réponde trait pour trait aux principes de catégorisation naturelle. Ces derniers sont l'objet de la psychologie cognitive, que la LC prend pour repère à partir des travaux de Rosh, en dépit de nombreuses insuffisances internes que nous explorerons plus loin ${ }^{6}$.

\section{Le modèle Roschéen}

2 A partir de quelques articles séminaux, Rosch a rouvert fort utilement le débat de la constitution des catégories partiellement oublié depuis les jeux de langage des recherches philosophiques de Wittgenstein. Le constat selon lequel les traditions universitaires faisaient des catégories des entités logiques bornées, quand les individus (sociaux) reconnaissaient des degrés de représentativité entre les membres des catégories, a conduit Rosch à procéder à des expérimentations visant à déterminer les principes de la catégorisation humaine. Aux méthodes « digitales » exclusives, qui définissent rigidement les traits constitutifs, s'opposent désormais les méthodes " analogiques », reconnaissant le caractère graduel de ces traits sur l'axe d'une même catégorie (Rosch et Mervis, 1975, p. 574). Toutefois, contrairement à ce qu'il est communément accepté, c'est relativement à la théorie définitoire de la psychologie, et non à un modèle philosophique général imputé à Aristote, que la théorie du prototype s'est développée (Pacherie, 1993, p. 2). Ainsi, la validation d'un concept était jusque là soumise à des conditions de satisfaction de contenu : pour posséder un concept, il fallait en connaître la définition, c'est-à-dire en posséder une représentation mentale qui satisfit une liste exhaustive de propriétés. Nulle place n'était laissée aux échelles de typicalité (prototypie). Il faut cependant reconnaitre, avant de marquer la nécessité d'un prolongement critique, qu'il s'agissait là de l'acquisition d'un concept public, intégré ou non (validé ou pas) au sein des représentations mentales d'un individu, en sorte que la popularité des travaux roschéens est en partie due à un malentendu initial ou à une ignorance épistémologique. Catégories, membres, concepts et représentations, dans leurs entrecroisements et multiplicités d'emploi obscurcissent souvent le propos. Là apparaît d'ailleurs, selon nous, une difficulté récurrente des études catégorielles: la caractérisation des objets d'études qui revêtent des acceptions et par là même des implicites bien différents selon les modèles. Ces variations (ou imprécisions) ont entre autres permis que d'un processus de vérification de traits, "légitime" si l'on distingue le concept public d'un réseau de représentations mentales, on bascule vers une procédure d'appariement consistant à ranger des catégories dans des taxonomies (cat, dog, turtle / animal). Si le concept public et le concept roschéen diffèrent, il n'en demeure pas moins que les modalités de leur constitution ont été considérées comme comparables.

Rosch ne reconnaît plus d'équivalence définitoire entre les catégories, puisqu'il est possible d'en sélectionner les meilleurs exemplaires (les prototypes). Cette asymétrie participe directement de l'organisation interne de la catégorie car le prototype sert de repère à la constitution d'un gradient (du plus représentatif au moins représentatif). Pour ce qui concerne les catégories physiologiques comme les couleurs, les formes, les expressions $\mathrm{du}$ visage, les prototypes (mentaux) correspondraient à des stimuli antérieurs à leur conceptualisation : les formes, couleurs et expressions faciales sont des traits saillants «naturels » déterminés hors des sujets. La plupart des prototypes sont toutefois dits postérieurs à leurs homologues naturels et les catégories sont principalement considérées comme des entités psychologiques acquises (au sens de l'apprentissage du sujet confronté aux réalités de son environnement). L'objectif de Rosch 
n'est pas pour autant d'isoler le principe qui sous-tend les stimuli et la formation des catégories : elle cherche à déterminer le système structural des catégories sémantiques. Pour être compris, les référents du mot n'ont pas besoin de posséder quelque trait commun qu'il soit. Ainsi conclut-elle, par l'appropriation de la notion de ressemblances de famille, à la relation graduée des référents entre eux (ibid.). Comme chez Wittgenstein, une structure catégorielle roshéenne peut donc être caractérisée par un ensemble de membres (avérés) qui ne partagent certains traits définitoires que localement ( $a b, b c$, de, ef etc.). Trois commentaires s'imposent déjà : le choix du mot comme unité d'appui tend à uniformiser et à restreindre la nature des objets et des classes constituées. Le mot est d'abord entendu comme un substantif, ce qui proscrit l'étude, ou même l'existence de catégories d'événements ou de processus (live, sing, laugh) par opposition à « l'état » du substantif, ce qui tendrait à révéler la nature figée du concept que l'on cible en réalité. Il est ensuite limité à son acception graphique (sofa, table, dresser pour la catégorie furniture, ou car, bus, truck, pour la catégorie vehicle) quand les unités symboliques sont des morphèmes ou des constructions dépassant leur simple graphie. Le mot enfin, que l'on accepte ou non son caractère référentiel, n'est pas son référent et particulièrement dans un contexte mentaliste de type LC, il conviendrait de les différencier (quoique nous soyons là déjà dans l'observation de l'implémentation contestable de ces résultats en $\mathrm{LC}$ ). Une conséquence importante de ces quelques remarques liminaires est la coupe qu'opèrent les choix méthodologiques sur les objets d'étude. Le mot, et le nom tout particulièrement, flècheront nécessairement des objets physiques ou psychologiques.

4 L'étude de Rosch s'intéresse en effet massivement à des objets concrets, des objets naturels ${ }^{7}$, qu'elle répartit selon une triple épaisseur d'abstraction: les niveaux élémentaire, superordonné et subordonné. Cet ordonnancement est conditionné par deux principes fondateurs qui déterminent les trois niveaux sur lesquels les catégories sont inscriptibles (Rosch, 1978, p. 29).

5 Le principe d'économie cognitive est un argument de bon sens, repris par Goldberg à propos du constructique. Il consiste en le rapport « économique » du système mental de traitement des informations à la somme d'informations extrinsèques données à percevoir (un argument du moindre effort pour soulager les ressources cognitives). Il s'agit de faciliter la comparaison des objets (les rapporter ou les opposer) à partir de l'anticipation de leurs traits totaux: prédire une appartenance catégorielle au seul moyen d'un trait donné ([+ tyres] pour car permet sa catégorisation anticipée $\left.e^{8}\right)$.

6 Le principe de réalité (perceived world structure) entend reconnaître un haut degré de corrélation entre la structure du monde réel et la structure du monde perçu auquel Rosch dit avoir accès au travers de ses expérimentations. Ces représentations internes constituent un ensemble structuré qui copient le monde et possèdent en conséquence nombre de ses attributs (ou traits) : ce sont les corrélats d'attributs. La cohésion ou l'incohésion des attributs de l'environnement sont alors reportées sur les connaissances du sujet, et parallèlement, sont institués dans la langue (Palmer, 1978).

7 La combinaison de ces deux principes a une incidence à la fois sur le niveau d'abstraction de la catégorie (dimension verticale) et sur sa structure interne (dimension horizontale). La dimension verticale est déterminée par le degré d'inclusion de la catégorie selon un axe graduel du plus spécifique au plus générique (collie, mammal, animal, living thing) et la dimension horizontale par la discrétisation de la catégorie à un même degré d'inclusion ( car, chair, wallet, pen etc.). Les deux principes ont ainsi pour double incidence : 
8 (1) que tous les niveaux de catégorisation ne sont plus utiles ; la largesse (imprécision) ou l'étroitesse (précision) du niveau n'intervient pas sur l'élémentarité de la catégorie.

(2) que la structure des catégories est asymétrique : il existe des prototypes constitués, au sens représentationnel fort, de traits plus représentatifs.

10 L'enjeu plus précis des expérimentations de Rosch consiste alors à valider le postulat selon lequel les taxonomies (les systèmes par lesquels les catégories sont liées entre elles) sont composées de catégories d'objets concrets (Rosch et al, 1976). Ces catégories, corrélats psychologiques d'unités cohésives du monde physique sont plus aisément constituées à un degré d'abstraction élémentaire (basic); les sujets, préférentiellement, discrétisent le monde à ce niveau plus accessible et plus immédiat. Les catégories ordonnées à niveau supérieur sur l'échelle de l'abstraction partagent seulement quelques traits quand les catégories subordonnées sont très redondantes (kitchen chair et livingroom chair partagent presque l'intégralité de leurs attributs). Par leur immédiateté conceptuelle, les objets du niveau «de base» correspondraient aux termes qui correspondent à des référents simples, ceux-là même qui sont intégrés prioritairement au cours de l'acquisition du langage. S'agissant du protocole, les phénomènes de typicalité ont été déterminés à partir des jugements émis par les sujets en fonction: du temps de catégorisation (plus court pour les exemplaires typiques), des erreurs de catégorisation (moins nombreuses pour les exemplaires typiques), de l'ordre d'apprentissage (les premiers exemplaires retenus sont dits plus typiques), de l'ordre de production (les exemplaires cités en premier) et des éléments utilisés comme points de référence (récurrence du choix de certains exemplaires comme points de référence).

11 De ce projet de description des procédés de catégorisation ressortent un ensemble de points centraux. Les catégories sont des dérivés d'objets concrets du monde (elles les représentent plus ou moins fidèlement). Elles constituent des taxonomies, forment des agrégats catégoriels partiels puisqu'elles partagent des attributs: les catégories sont reliées par ces traits communs ( $a b, b c$, de etc.), si bien qu'il est possible de comparer leur degré de similitude sur trois axes d'abstraction plus ou moins médiats. Le niveau élémentaire (ou basique) est l'axe privilégié le long duquel les catégories sont le plus similaires. A un niveau de description commun, les catégories sont radialement représentables selon des variables de typicalité.

\section{Quelques problèmes}

12 A l'encontre de ces traits théoriques centraux peuvent être portées de nombreuses critiques. Toutes ont une incidence médiate sur la légitimation « de bon droit » proposée par la LC et attendu par son engagement empiriste. D'abord, se fixer pour objectif la description du réel (perçu) pose le problème génétique (au sens de l'origine) de la possibilité d'un accès au monde. Si l'on considère valide la thèse des structures corrélationnelles entre le monde et l'individu, l'entreprise de Rosch vise à décrire le savoir. Or, les traits isolés qui permettent de comparer les catégories entre elles dans les taxonomies sont des traits " constitutifs ». Il faudrait alors que le savoir soit constant, et non labile, ce qui pose de fait une inadéquation du modèle relativement à ses aspirations. Ensuite, cette représentation de l'environnement et de ses possibles contiguïtés se fait à partir de relations d'inclusion, qui ne constituent qu'un aspect de l'organisation des connaissances. Markman (1983) et Carbonnel (1978) montrent en effet que les relations de 
métonymie ou de collection sont plus précoces que les relations d'inclusion (arbre/forêt, soldat/armée), ce qui tend à invalider les résultats de Rosch du point de vue de leur application à l'apprentissage et à l'acquisition des connaissances (chez l'enfant). En anthropologie, Levi-Strauss (1962, p. 85) voulait déjà reconnaître des formes de connaissances non typologiques (c'est-à-dire dénuées de « traits » constitutifs) à partir de contiguïtés perçues dans l'expérience (serpent/termitière, fourmi rouge/cobra [rouge]), qui plus est non fondées sur des taxonomies biologiques (Dubois, 1991, p. 36). Dubois attribue en effet une large part de l'inefficacité du modèle proposé par Rosch à l'universalité présumée de l'organisation cognitive sur laquelle il repose, ce «biais du projet épistémologique de la culture occidentale à vouloir établir des classes d'objets strictement naturels » (ibid.).

13 Il faut aussi prendre en compte un certain nombre de confusions, assez tôt remarquées, qui jettent le discrédit sur ces travaux de la psychologie. Une première confusion concerne les notions d'appartenance catégorielle et de représentativité qui sont ici assimilées. Or, l'appartenance n'est pas une question de degrés. Armstrong et Gleitman (1983) ont montré que la catégorie des nombres impairs était sujette à des effets de typicalité. 3 et 7 sont plus typiques que 61 mais n'en sont pas pour autant plus impairs. De même ostrich et swallow exhibent des degrés de typicalité différents mais appartiennent à une même classe. Ainsi pouvons-nous conclure à l'asymétrie des phénomènes de typicalité et de la structure du concept.

14 Nous relevions plus tôt le choix restrictif du mot comme outil de caractérisation. Au delà encore du caractère statique (état, nominalité) des objets, les résultats obtenus à partir des catégories d'espèces naturelles ont été étendus aux artefacts sans plus de rigueur explicative. Or, par la corrélation entre les traits du concept et les attributs du monde, ce qui caractérise un concept d'objet naturel consiste en un ensemble de propriétés perceptives. On est alors bien loin des traits fonctionnels des artefacts jugés plus importants ou plus typiques que des traits perceptifs « définitoires » (chair possèderait un trait fonctionnel [to have a seat], et non définitionnel [four feet]). A l'inverse, Rosch relève des taxonomies floues au sein même des espèces naturelles à partir des multiples hésitations des sujets (fruit/legume [tomato]). Or, ces catégories dépendent de pratiques humaines et sont en cela éminemment contextuelles, ce que les hésitations révèlent certainement. La frontière "floue » séparant les deux catégories est donc fictive, elle marque l'incapacité du sujet à classer une notion hors de son contexte d'emploi. La création d'un flou central dans un domaine topologique est une solution de repli sans égards pour la multiplicité des concepts humains.

\section{Un modèle scolastique?}

15 Toutes ces critiques internes sont en somme rapportables au simple fait de caractériser les catégories par des traits définitoires, comme dans les méthodes « digitales » ou fixistes contre lesquelles Rosch veut pourtant ériger son modèle. Conséquemment, l'appartenance catégorielle est validée par le degré de similitude avec le prototype (un exemplaire constitué des meilleurs attributs) alors que rien n'est dit de la labilité (contextuelle) de ces attributs et de leur possible nature non constitutive (ces attributs ne sont pas des «propriétés constitutives »). Enfin, leur pertinence et leur sélection restent inexpliquées. De quels critères dépendent les traits « saillants » ? Murphy et Medin (1985) font de la comparaison un argument de poids contre la théorie du prototype. Plum et 
lawnmower partagent un ensemble de traits: l'une et l'autre peuvent être déplacées, ne voient pas, ne sont pas capables de sentiments, etc. La similitude entre deux catégories peut s'avérer arbitrairement grande ou petite. Sauf à pouvoir donner des propriétés objectives (ce qui est impossible), la théorie roschéenne de la catégorisation est erronée. Il est d'ailleurs surprenant de voir dans la littérature l'opposition systématique entre Aristote et Rosch, car ce qui limite la validité de la théorie du prototype est son recours à des conditions de contenu tout comme dans la théorie scholastique. La comparaison est également instructive et révélatrice lorsque l'on considère les plans (ou les niveaux d'abstractions) des deux théories. Sur le plan vertical où sont généralement représentés les trois niveaux roschéens, le niveau élémentaire correspond à l'espèce et le niveau surordonné au genre des prédicaments d'Aristote ( $2^{\text {nd }}$ analytiques, $\left.90 \mathrm{~b}\right)$. Ces prédicaments sont les différentes manières dont les catégories peuvent être les prédicats d'un sujet. Porphyre en distingue cinq (le genre, l'espèce, la différence, le propre et l'accident) quand Rosch les scinde en deux groupes, le genre et l'espèce qui situent les objets dans les catégories et la différence qui définit les objets à l'intérieur des espèces. Elle ne traite donc ni du propre, ni de l'accident, ce qui la pousse à indifférencier la variété des catégories (ou des types catégoriels) et à recourir à des conditions nécessaires et suffisantes. On ne peut pas, dans ce système, différencier par exemple, les sous-types de feuilles, ni les sous-types de branches. Cela n'est pas nécessaire parce que les éléments du niveau de base, c'est-à-dire de l'espèce, sont donnés et saisis intuitivement à un degré d'abstraction qui les discrimine. La perception est alors passive, comme le suggérait déjà Thomas d'Aquin dans De veritate en distinguant le discursus de l'apprehensio simplex, les articulations de la deuxième opération de l'esprit ${ }^{9}$. D'autres ont remarqué ces insuffisances. Rastier (1991, p. 278) dit de la théorie roschéenne qu'elle est « une variante appauvrie de la conception aristotélicienne», quand Pacherie (in Dubois, 1991) parle ironiquement de « ressemblances de famille » entre Rosch et Aristote en soulignant les simplifications faites par Rosch en abordant la position définitoire scholastique.

\section{Prototypes psychologiques et sciences cognitives}

16 Plusieurs études parallèles en psychologie du développement, neuro-psychologie et ethnologie, tendent aussi à souligner les insuffisances mentionnées plus haut et en particulier l'hétérogénéité des facteurs entrant en compte dans les processus de formation des catégories.

17 Keil et al (1986) montrent que les enfants sont, très tôt, capables de distinguer les espèces naturelles (trees, fruits, fish, birds) des objets artificiels, ou "artefacts» (tools, clothes, furniture, vehicles). Ils attribuent des propriétés constantes aux seules espèces naturelles quand les traits des artefacts varient systématiquement: une cafetière qui fait office de mangeoire à oiseaux ne possède plus les traits de la cafetière dès lors qu'est opéré le changement de fonction.

18 Hart et al (1985) suggèrent que certaines lésions cérébrales interfèrent sur les choix d'inclusion ou d'exclusion, cela voulant indiquer la localisation d'un champ gnostique, une " aire de la connaissance ». Sans aller jusque-là, on pourra reconnaître l'intérêt de cette étude qui repose principalement sur la possibilité envisagée d'un système de reconnaissance (visuelle et verbale) différencié pour les espèces vivantes et les artefacts. Impossible alors d'étendre les principes catégoriels de l'un à ceux de l'autre. 
19 Atran (1990), enfin, dresse le bilan de résultats en ethnologie tendant à montrer qu'au delà de la diversité des cultures (Zafimaniri de Madagascar et Hanunoo des Philippines) les catégories d'espèces naturelles sont comparables, quoique non strictement similaires. Ainsi faudrait-il marquer une distinction entre les espèces naturelles qui donnent lieu à des constantes (relatives) de catégorisation et les artefacts, changeant du tout au tout. Les principes établis par Rosch à partir des catégories "perceptives", constituées et observées dans leur rapport à des objets concrets relevant d'espèces naturelles, ne peuvent pas être étendus à l'ensemble des catégories. La question du langage (en tant qu'ensemble d'opérations révélées au travers des langues) ou des langues (en tant qu'ensemble de signes abstraits de l'usage) devient alors on ne peut plus problématique. Selon que l'on considère l'un ou l'autre l'objet de la linguistique, les principes de catégorisation naturelle (déjà peu valides) touchent des domaines différents ; individuel et cognitif d'une part, puis collectif et culturel d'autre part. Même à considérer l'incidence de l'individuel sur le collectif, l'étude du sens ne peut pas être réduite au système d'organisation des connaissances des locuteurs. La sémantique (en tant que discipline) ne peut pas consister en la seule étude du lexique mental du sujet cognitif, or le constructique correspond à un ensemble de signes ordonnés qui implique que l'acte de langage consiste en un partage (et non en une construction spontanée) de connaissances fondées par des expériences quotidiennes. Elles sont de plus identiques dans les représentations mentales de tous les individus ${ }^{10}$ et ce au mépris des relativismes culturels. Une des raisons que nous invoquons pour expliquer le centralisme de la LC sur les structures cognitives des sujets est l'appropriation non critique des principes probabilistes de Rosch pour rendre compte des structures sémantiques. Le fait d'inscrire le linguistique (et la linguistique) dans les sciences cognitives en tant qu'il jouerait le rôle de fenêtre ouverte sur la cognition est un parti-pris théorique, mais ouvrir une fenêtre sur la cognition n'implique ni la bijectivité, ni l'indépendance des structures conceptuelles et sémantiques. En d'autres termes, le signe (et plus largement, le système symbolique) est-il le fait des seuls sujets cognitifs ou peut-il être distribué ${ }^{11}$ ? Loin de ces interrogations, la LC a étendu aux sens des mots les principes de la catégorisation naturelle comme Rosch avait étendu les principes des catégories naturelles aux catégories artificielles.

\section{Les autres théories du concept?}

La catégorisation est essentielle à toute l'architecture de la LC qui vise, au travers elle, la détermination empirique du concept pour appréhender la part sémantique du langage. Pourtant, prendre pour épicentre les méthodes « avérées » de la catégorisation implique que l'on puisse les déterminer. Or, force est de constater que les méthodes roschéennes sont discutables. En outre, par son attachement au prototype, la LC s'efforce de rendre compte de la structure des concepts (ou de leur formation) mais ne montre pas en quoi cette structuration est pertinente pour l'analyse linguistique. Bien sûr, il n'y a pas lieu de différencier concepts et sens ou de parler de la nature de leur rapport (puisqu'ils sont dits isomorphes) mais rien n'est dit du rôle de l'activité langagière dans la création de ces repères conceptuels "partagés» (selon un processus tout aussi tu). La psychologie et la linguistique n'ont plus ici distingué leurs objets, partant d'un constat identique (l'insuffisance du modèle scholastique) bien qu'elles aient reproché à Aristote des aspects différents de sa théorie du concept. La psychologie (Rosch, 1978, p. 29) 
refusait qu'elle laissât aux facteurs socio-historiques une possibilité explicative (les catégories, quelles qu'elles soient, sont le fruit de principes humains: la lecture de l'individu sur le monde) quand la linguistique (Lakoff, 1987, p. 6) reprochait à Aristote son objectivisme (les choses qui «existent» dans le monde hors des phénomènes de perception). On s'étonnera, compte tenu l'importance de la catégorisation, que la LC ait décrié le modèle catégoriel classique pour les raisons inverses de celles invoquées par Rosch (arbitrarité catégorielle et non objectivisme) tout en l'intégrant en tant que théorie fondatrice. Lakoff, toutefois, aura voulu marquer son originalité et se distancer de Rosch, non par le rejet de la notion de prototype (l'empirisme des études psychologique servent l'engagement cognitif de la LC) mais par l'élaboration des modèles cognitifs idéalisés. Pour une raison différente des critiques que nous avons mentionnées plus haut (et qui leur est d'ailleurs conséquente), Lakoff propose une théorie transformationnelle des représentations mentales dans une veine comparable au mentalisme chomskyen. Les effets de typicalité, on l'a vu, consistent en la reconnaissance empirique, c'est-à-dire "constatée » par Rosch à partir des intuitions des sujets interrogés, de meilleurs exemplaires au sein des catégories. Cette évidence empirique à la fois fâcheuse et incontestable doit être contournée: c'est la raison pour laquelle Lakoff recourt à des modèles cognitifs stabilisés (mentaux) dont les effets de typicalité ne sont que la surface ou les symptômes (réalisation linguistique). Les résultats de Rosch sont préservés, mais ils ne seraient que la manifestation d'agrégats ou de conjonctions de cadres (frames) internalisés, c'est-à-dire des théories mentales articulées ou des connaissances idéalisées à propos de l'état ou du fonctionnement du monde. On retrouvera l'ensemble des traits de l'approche lakoffienne de la catégorisation dans la theory theory (Carey, 1985 ; Murphy et Medin, 1985 ; Gopnik et Meltzoff, 1997) sans qu'il ne soit, par ailleurs, cité en retour. Quoiqu'il en soit, on peut imputer une large part des critiques émises à la théorie du prototype au cumul ponctuel de traits contradictoires extraits de différents modèles idéalisés. L'exemple classique, parangon de la théorie scholastique, peut ainsi être traité sans que l'on ait à recourir aux traits définitoires, ni aux effets de typicalité (dont on ne peut dire plus que leur incohérence avec les choses du monde). Bachelor est ainsi dit être relié à un ICM (idealized cognitive model) qui inclut des informations larges, culturellement stabilisées et possiblement mêlées : une société monogame, le mariage et ses institutions, l'âge standard des époux etc. Dans ce cadre idéalisé, il est normal que l'occurrence pope paraisse être un mauvais exemple, car il est aussi lié à l'ICM qui rend compte des hiérarchies et des lois au sein de l'Eglise Catholique et à l'intérieur duquel il est «typique » de ne pas se marier.

21 Les effets de typicalité peuvent toutefois, à l'inverse, être le fruit d'agrégats plus complexes à partir, cette fois, de traits convergents. Ainsi, mother peut renvoyer à plusieurs de ces modèles ou ensembles culturaux qui composent les classes et sous-classes mentales de référence en fonction desquelles les catégories sont constituées (birth model, genetic model, nurturance model, marital model, genalogical model). Selon que le sujet insiste sur les traits idéaux des différents modèles, il se réfère à une sous-classe au travers de laquelle il accède aux autres. Les effets de typicalité viennent alors du fait de la sélection d'un sous-modèle en tant que meilleur modèle ; un modèle plus prompt à s'insérer dans le contexte. L'ICM contre lequel est repéré mother est donc multiple et les scènes mentales figées qui le sous-tendent sont inégalement convoquées. Le sujet cognitif fait appel au modèle natal dans lequel la mère est celle qui donne naissance face à des contextes de type " adoption " (I don't know who my real mother is), au modèle génétique lorsqu'il est 
question de la naissance hors de l'affect (My real mother died when I was an embryo) et ainsi de suite pour chaque sous-catégorie. La sélection d'une sous-classe (birth, genetic, marital etc.) entraine l'organisation de toute la classe (mother), en fonction du meilleur exemplaire, choisi suivant le contexte et soumis pour validation ou invalidation aux propriétés d'un ICM plus ou moins complexe. A l'inverse de la théorie du prototype standard, on n'exclut plus un objet d'une catégorie en fonction des traits de cet objet, on le rapporte aux conditions idéalisées des cadres ou des espaces mentaux.

22 Les effets de typicalité sont aussi expliqués en termes de "métonymie » (au détriment d'ailleurs de l'acception stricte de cette figure) ; un exemplaire peut incarner la catégorie entière (Downing street refused comment, I am parked out the back) au détriment des normes ou des attentes véri-conditionnelles. Les autres membres de la catégorie sont alors, de fait, relégués au rang de mauvais exemplaires. Ces exemplaires métonymiques peuvent être sociaux, idéaux, parangoniques etc., puisque le choix d'isoler, d'individuer un membre pour représenter un ensemble revient au seul sujet qui compose en fonction de ses modèles mentaux. En somme, si les ICM permettent de préserver les observations de Rosch tout en étant une théorie adéquate de la représentation des connaissances, c'est avant tout parce qu'elle ne diffère qu'en un seul trait de la théorie du prototype : la relégation des effets de typicalité au sujet cognitif, autrement dit la caractérisation non plus absolue des objets (au sens large) mais contre des scripts cognitifs plus ou moins culturellement partagés. A cette seule exception, l'ensemble de nos critiques garde sa pertinence et se retrouve disséminé dans l'application sémantique de ces théories catégorielles.

23 En dépit de cette relecture de l'esprit en tant qu'interface d'activation de représentations mentales, on s'étonnera que la LC ne se soit pas intéressée à d'autres modèles catégoriels et, à l'inverse, qu'elle n'ait pas séduit les psychologues ou anthropologues attachés aux mêmes problématiques. Comme elle l'a fait à l'encontre de la grammaire générative (dont elle voulait se séparer depuis la sémantique générative), la LC semble s'être concentrée sur un point de comparaison exclusif, entretenant un rapport contradictoire avec les principes catégoriels qu'elle voulait critiquer. Il est par ailleurs assez communément admis hors de la linguistique (Laurence et Margolis, 1999) que la famille des théories catégorielles s'étend à cinq, chacune de ces théories étant différenciée en fonction du rapport qu'elles permettent entre les membres d'une catégorie de concepts (structure définitoire, structure probabiliste, structure théorique, sans structure, structure plurielle). De plus, si toutes partent du constat négatif de l'impropriété de la structure définitoire classique, toutes ont aussi reconnu les insuffisances du modèle prototypique roschéen que la LC entretient au risque d'habilitations périlleuses. Aux traditionnelles méthodes scholastique et probabiliste (classique et prototypique) se sont par exemple superposées, l'atomisme (Fodor, 1998; Millikan, 2000) et le pluralisme conceptuel (Laurence et Margolies, 1999) qui apportent des solutions différentes aux problèmes généraux de la représentation des connaissances.

\section{Catégorisation des objets sémantiques}

24 La LC persiste à considérer avérés les résultats de l'analyse roschéenne de la catégorisation humaine quoiqu'elle soit confrontée aux difficultés de la typicalité des exemplaires. La LC propose cependant un contournement élégant de ces difficultés par la relativité mesurée de ses effets qu'elle attribue à divers mécanismes d'articulation entre 
les scènes mentales figées dont dispose le sujet et ses capacités de ciblage et de comparaison qu'il convoque en situation. Toutefois, mise à part la nature du lien entre les membres, les propriétés du modèle prototypique restent valables, tout comme les insuffisances identifiées plus haut. En premier lieu, il nous semble que les principes identifiés au cours de la description des catégories perceptives sont inadéquats à rendre compte des catégories artificielles et plus encore des catégories sémantiques.

Il ne va pas de soi que les étapes successives observées dans la constitution perceptive des catégories soient aptes à décrire des extensions grammaticales et ce que chacune constitue une catégorie ou non. Cette iniquité repose en partie tout au moins dans la définition même du vocable. Est-il bien certain que les disciplines linguistique et psychologique, même connexes, aient abordé le même objet? Comme mentionné supra, les catégories sont des représentations d'objets naturels qui obéissent à des règles de hiérarchisation et de taxinomie (dimensions verticale et horizontale) et non des items lexicaux dont on voudra déterminer la multiplicité des sens.

D'abord, les niveaux d'abstraction et les degrés de l'analyse sémantique ne sont pas congruents. Dans la langue, les termes communs sont tout aussi sujets aux variations que les hyperonymes et les hyponymes : food, cake et carrot cheese cake ne sont-ils pas tous trois de bons candidats à l'observation de leurs variables de sens (extension par analogie, sens «figurés», etc.) ? Le degré de complexité n'entame pas l'intégrité des objets (sémantiques). La restriction de Rosch au niveau de base limiterait donc l'étude sémantique à des réseaux de sens issus de termes communs, discriminés par les sujets sur la base de leur immédiateté. Toutefois, si l'on passe outre cette restriction, on se sépare de fait d'une caution empirique: la LC réasserte pourtant son attachement à cette condition empirique au travers de son engagement cognitif.

A ce niveau élémentaire (ou " de base »), qui est un niveau d'abstraction méthodologique et non une sous-classe lexicale, s'équilibrent les principes de réalité et d'économie cognitive. Nous avons longuement commenté le premier et voulons ici plus encore souligner l'inanité du second (économie cognitive). La phonétique historique a rejeté les lois du moindre effort, reconnaissant la précellence de l'intelligibilité dans les processus d'évolution du latin vers le français (coupe diachronique), comme à l'intérieur même des langues contemporaines (coupe synchronique) (Lüdke, 1986). Certes, il est possible d'identifier un patron récurrent qui voudrait incarner cette loi: le morphème « aujourd'hui », gallicisé à partir du latin hodie a d'abord subi une usure phonétique pour devenir hui. Par amplification dite sémantactique, il s'est ensuite étendu à un syntagme nominal (au jour d'hui, quatre unités, ou mots graphiques) pour s'agglutiner (aujourd'hui) et se trouver à nouveau sujet à la subduction. On peut donc identifier un schéma de type : usure phonétique, amplification sémantactique, fusion et usure de nouveau, ce de façon circulaire. Toutefois, si les lénitions et les pertes de segments initiales sont motivées par une possible loi du moindre effort au niveau phonétique, il n'en est déjà plus rien lors de l'amplification sémantactique, car il s'agit d'une adjonction de matière lexicale pour assurer la bonne transmission du message (ou du moins permettre sa co-construction en situation). Le processus est aussi éminemment créatif, puisque le principe de fusion s'accompagne d'extensions (repère temporel immédiat, ou ère plus ou moins localisée ${ }^{12}$ ). En somme, il n'est pas lieu de penser que ce processus soit sous-tendu par une "fuite " vers la facilité expressive, sauf à considérer que la créativité et l'adjonction lexicale convoquent des ressources cognitives plus simples. Par ailleurs, comme le souligne Keller (1994, p. 150), l'acte de communication est « une petite expérience sociale», au cours de 
laquelle les sujets qui interagissent vérifient à mesure l'état de l'interaction dans laquelle ils sont engagés. Chacun y introduit des modifications en fonction des interlocuteurs ( feed-back). Une langue est constituée pour qu'elle fonctionne en tant que système homoérhétique sans qu'il y ait même de tension entre ses logiques de fonctionnement et de constitution. Par cette inscription sociale, la variation linguistique est motivée par une dynamique d'interprétation et non par une loi du moindre effort. Bien sûr, la thématisation permet un accès plus immédiat à l'unité de sens (quoique les contextes qui sont construits et reconstruits appellent un ajustement systématique) mais la dynamique générale du changement linguistique est plus complexe qu'une simple lénition. Par ailleurs, les tests de fréquence à partir des registres de langue montrent que l'économie syntaxique est inopérante : " est-ce que » est très largement préféré à l'inversion sujet/ verbe, plus soutenue mais plus économe du point de vue des réalisations linguistiques et des exigences phonatoires qu'elles appellent (Lapaire et Rotgé, 1991).

De plus, si le niveau de base en psychologie connaît, semble-t-il, une vague correspondance linguistique, il correspond plutôt à un choix préférentiel, adaptable en fonction de niveaux d'expertise (Kleiber, 1988, p. 35). Ceci fait écho aux catégories 'ad hoc' de Barsalou (1993); elles sont constituées localement et dynamiquement, sont ensuite utilisées comme point de repère dans un contexte spécifique et n'ont de pertinence que relativement à ce contexte (par exemple la catégorie what to take from one's home during a fire). Ainsi, le niveau de base, qui ne voudrait comprendre que les prototypes peut inclure des lexèmes centraux de complexité variable (surnumérariats, enceintes de monitoring, U.V., etc.) qui appartiendraient à des niveaux d'abstraction différents selon la classification roschéenne. Pour que la théorie de la catégorisation soit opératoire en linguistique, il faudrait que le lexique des langues (ou celui des noms, puisque Rosch ne s'intéresse qu'à lui) soit organisé comme une taxonomie. Cependant, à l'exception ponctuelle du vocabulaire technique (propre à la classification linnéenne) et de certaines régions lexicales des sciences naturelles, l'organisation des structures lexicales diffèrent des taxonomies conceptuelles.

Il n'est en somme pas surprenant que la théorie du prototype ne se soit pas risquée à définir les classes lexicales au travers des langues (typologie) d'une part, et au travers de l'histoire d'une langue d'autre part (diachronie), car les phénomènes de catégorisation spontanées qui permettent (en toute partialité) de déterminer les catégories perceptives n'ont aucune emprise analytique sur l'histoire des classes lexicales. L'universalité et l'immédiateté ${ }^{13}$ des principes prototypiques en sont à l'évidence responsables et nous devons ajouter à cela l'incapacité explicative de ces modèles à traiter des occurrences et de leur(s) classe(s) dans une langue donnée, même en synchronie ${ }^{14}$. Rastier (2000) reconnait des inégalités qualitatives entre les classes lexicales en distinguant deux types de prototypes panchroniques : les termes à usage générique (valorisation neutre) et les parangons (valorisation positive). Plaçant au premier plan les variations des normes de valorisation ${ }^{15}$, il montre l'incidence de la mélioration dans la constitution des usages et plus largement celle des normes culturelles sur la langue et les éléments saillants de cette langue. Cet angle d'approche, comme Hjelmslev et Leibnitz auparavant (Hjelmslev, 1971, p. $164 ; 1985$, p. 34 ; Leibnitz, NEEH, IV), insiste sur le caractère intensif ou extensif, non des entités référées, mais des items lexicaux : soit homme et femme; homme est extense, il renvoie à la catégorie entière ou à un exemplaire de cette catégorie, femme est intense car il est l'autre membre de l'ensemble sans pouvoir représenter cet ensemble. On pourra dire en d'autres termes que femme entretient un rapport métonymique avec la catégorie 
homme quand l'exemplaire homme lui est isomorphe. Homme est ainsi caractérisé par deux acceptions (homme vs animal; homme vs femme). Ainsi est-il possible de constater un état de la langue, le rapport métonymique de deux unités lexicales, sans postuler ce même rapport entre les entités référées (ici hautement problématique).

La théorie du prototype, bastion principiel de la LC, a pour principal avantage de poser (au travers de Rosch) la question des inégalités qualitatives au sein des classes lexicales. Cependant, le prototype - sa définition et ses incidences sur l'organisation de la catégorie, sa gradualité - diffère du tout au tout selon les modèles, en sorte qu'il est parfois difficile d'identifier le modèle auquel recourt l'auteur ${ }^{16}$. Aux zones d'ombres théoriques s'ajoutent les blancs explicatifs. Face aux exigences de la diachronie, le prototype s'avère partiel : il ne peut pourtant pas (en langue) être achronique sauf à le considérer immuable et naturel, ce que Rosch postule implicitement des objets concrets. Nous constatons cependant avec Rastier (ibid., p. 162) que « mammouth n'est plus le prototype de gros gibier ». Il faut bien reconnaître que la LC, fidèle aux principes roschéens, ne se pose pas la question de la fixité ou de l'instabilité des prototypes au travers des contextes et des états de la langue. L'une des raisons que nous avons proposées plus tôt, en abordant le rapport postulé des catégories naturelles aux catégories linguistiques, semble ici se confirmer. Si l'on considère comme Rosch initialement que le prototype est naturel, unique et universel ${ }^{17}$, il n'y a pas lieu de s'interroger sur les fluctuations culturelles ou diachroniques des prototypes. En ne précisant pas quel est son objet (concret « perçu » ou symbolique), la LC importe des implicites avec lesquels elle ne peut pas être en accord ${ }^{18}$.

Outre cet ensemble d'inadéquations, et bien que la psychologie cognitive et la LC se recommandent l'une l'autre à partir de buts et d'observations communs, chacune aborde son objet à l'inverse de l'autre. La psychologie aborde le concept au travers du mot, quand la linguistique en décrit les acceptions, et en particulier les classes d'acceptions. A l'émergence (la catégorisation) spontanée d'un concept (onomasiologie) ne correspond pas le signe, son histoire, ses contextes d'emploi, ses registres etc. S'il est déjà contestable qu'un concept identifié en psychologie corresponde à un objet du monde, ou même à un objet mental, conceptualisé de ce monde, que dire d'une unité linguistique? A un mot ne correspond jamais un signifié et organiser une catégorie des sens du mot permet, en effet, d'outrepasser ce préjugé de la philosophie du langage ; mais il ne s'agit là que d'un choix par défaut qui, de plus, permet la polysémie partielle de ces acceptions de mot quand chacune appartient à des discours ou à des textes (en somme à des contextes) de nature variable (voiture, automobile, bagnole, caisse, guimbarde, tacos etc.).

32 Parce que la LC recourt à l'articulation des connaissances pour lier l'esprit et la langue, il est souvent difficile d'identifier la source réelle des problèmes qu'elle entend résoudre. Avant Saussure, la tradition occidentale avait aussi très systématiquement lié les théories du signe et les théories de la connaissance. Les nominalistes et les empiristes en sont seulement deux exemples. Condillac rapporte la grammaire à l'art de bien penser et les sciences à des langues «bien faites ». Les études ockhamistes s'appuient en particulier sur trois fondements similaires aux positions du réalisme incarné : la distinction entre connaissance intuitive et connaissance abstractive, l'idée générale de langage mental, puis l'idée selon laquelle les concepts sont à la fois des actes intellectuels et des signes (Panaccio, 2005, p. 423). Dans les linguistiques de la cognition (grammaire générative et cognitive), la problématique est la même : relier l'un et l'autre des extrêmes supposés du sens (la connaissance, logique ou phénoménologique, et le produit linguistique). Mais le versant psychologique de la sémantique cognitive s'avère infondé en raison du caractère 
versatile des objets de la psychologie et de la linguistique qui, non seulement diffèrent en nature, mais ne sont pas abordés depuis le même angle méthodologique. Nos recherches nous ont conduit à mettre en avant l'existence d'un implicite problématique: l'appariement injustifié des classes de concepts et des classes linguistiques. La sémantique $\mathrm{du}$ prototype demeure cependant très influente, en dépit du flou des outils analytiques qu'elle fournit aux linguistes ou aux psychologues, et du flou et de la variabilité des résultats attendus.

\section{BIBLIOGRAPHIE}

\section{Références}

ARMSTRONG, S., L.R. Gleitman et H. Gleitman. 1983. « What some concepts

might not be ». Cognition, $13: 263-308$

ATRAN, SC.1990. Cognitive Foundations of Natural History. Cambridge:

CUP.

BAILLARGEON, R. 1999. « Young infants' expectations about hidden objects :

a reply to three challenges ». Developmental Science, 2 (2) : 115-163.

BARSALOU, L.W. 1999. «Perceptual symbol systems ». Behavioral and Brain

Sciences, $22: 577-609$.

BARSALOU, L.W., W. Yeh, B.J. Luka, K.L. Olseth, K.S. Mix et L.-L.Wu.

1993. «Concepts and Meaning ». En ligne : www.psychology.

memory.edu/cognition.

BUSH, G., L. Phan et Michael I. Posner. 2000. « Cognitive and Emotional

Influences in Anterior Cingulate Cortex ». Trends in Cognitive Sciences, 4 : 215-222.

СномSку, N. 1957. Syntactic Structures. Gravenhage : Mouton.

СНОмSку, N. 1980. Rules and Representations. Oxford : Blackwell.

CHOMSкY, N. 1981. Lectures on Government and Binding. Dordrecht,

Holland, Cinnaminson : Foris Publications.

COHEN, L. B. 1995. « How solid is infants' understanding of solidity ». Paper

presented at the Society for Research in Child Development. Indianapolis, March.

CORRIGAN, R. (ed.) 1989. Linguistic Categorization. Amsterdam : John

Benjamins. 
DUBoIs, D. (ed.) 1997. Catégorisation et cognition. Paris : Kimé.

DUBoIs, D. 2006 « De l'expérience subjective des catégories de couleurs à

l'objectivité de la couleur : approches cognitives ». Cahiers du LCPE,

$\mathrm{n}^{\circ} 7$ : Dénomination, désignation, catégories : 67-78.

DURAND, K. 1999. « La représentation d'une représentation. Le passage de 3 à

2 dimensions chez les bébés de 3 et 4 mois ». Thèse de doctorat en

psychologie, Université René-Descartes.

FAUCONNIER, G. 1984. Les espaces mentaux. Paris : Les éditions de minuit.

FAUCONNIER, G. 1997. Mappings in Thought and Language. Cambridge :

Cambridge University Press.

FODOR, J. A. 1998. Concepts: Where Cognitive Science Went Wrong. New

York : Oxford University Press

GARCEAU, B. 1968. Judicium. Vocabulaire, sources, doctrine de Saint Thomas

d'Aquin. Paris : Vrin.

GOLDBERG, A. 1995. Constructions: a Construction Grammar Approach to

Argument Structure. Chicago : University of Chicago Press.

GOLDBERG, A. 2003. «Constructions: a New Theoretical Approach to

Language ». Trends in Cognitive Sciences 7 (5) : 219-224.

GOLDBERG, A. 2006. Constructions at Work : the Nature of Generalization in

Language. Oxford: Oxford University Press.

GUIGNARD, J.-B. 2010. « Sémiotique cognitive - aspects d'une autre théorie

computationnelle ». Texto, $\mathrm{XV}, 1$.

HAITH, M. M. 1998. « Who put the cog in infant cognition? Is rich

interpretation too costly? », Infant Behavior and Development, 21 (2) :

167-179.

HART, J. et R. Berndt. 1985. « Category-specific naming deficit following

infarction », Nature : 316-439.

HJELMSLEV, L. 1942. « Langue et parole ». Cahiers Ferdinand de Saussure,

$1942(2)$.

HJELMSLEV, L. 1971. Prolégomènes à une théorie du langage. Paris : Éditions de Minuit.

JACKENDOFF, R. 1983. Semantics and Cognition. Cambridge, Mass. : MIT

Press.

JACKENDOFF, R. 1990. Semantic Structures. Cambridge, Mass. : MIT Press.

JOHNSON, C. 1999. «Constructional grounding: The role of interpretational 
overlap in lexical and constructional acquisition ». Thèse de doctorat. Université de Californie, Berkeley.

KELLER, R. 1994. On Language Change: The Invisible Hand in Language.

London : Routledge.

KLEIBER, G. 1988. « Prototype, stéréotype : un air de famille ?» DRLAV, 38 :

$1-66$.

KLEIBER, G. 1990. La sémantique du prototype. Paris : PUF.

LAKOFF, G. 1987. Women, Fire, and Dangerous Things: What Categories

Reveal about the Mind. Chicago : University of Chicago Press.

LAKOFF, G. et M. Johnson. 1980. Metaphors We Live By. Chicago :

University of Chicago Press.

LAKOFF, G. et M. Johnson. 1999. Philosophy in the Flesh: the Embodied Mind

and its Challenge to Western Thought. New York : Basic Books.

LANGACKER, R. 1987. Foundations of Cognitive Grammar, Vol. 1, Theoretical

Prerequisites. Stanford : Stanford University Press.

LANGACKER, R. 1991. Foundations of Cognitive Grammar, Vol. 2, Descriptive

Application. Stanford : Stanford University Press.

LAPAIRE, J.-R. et. W. Rotgé. 1991. Linguistique et grammaire de l'anglais. Toulouse

: Presses Universitaires du Mirail.

LAURENCE, S et E. Margolis. 1999. Concepts. Blackwell Guide to the

Philosophy of Mind.

LECUYER, R. « Rien n'est jamais acquis ou de la permanence de l'objet... de

polémiques ». Enfance, 2001/1:53.

LEVI-STRAUSS, C. 1962. La pensée sauvage. Paris : Plon.

LOCKE, J. 1690[1997]. An Essay Concerning Human Understanding. London,

Penguin.

LÜDKE, H. 1986. « Esquisse d'une théorie du changement langagier ». La

linguistique $22: 3-46$.

MARKMAN, A.B. 1999. Knowledge Representation. Mahwah : Laurence

Erlbaum Associates.

MARKMAN, E.M. 1983. « Two different kinds of hierachical organization ».

New Trends in Conceptual Representation : 164-184.

MICHAELIS Laura A. 2006. « Construction Grammar ». In K. Brown, Editor-

in-Chief, Encyclopedia of Language and Linguistics, Second Edition,

volume 3, 73-84. Oxford : Elsevier. 
MORGENSTERN A., Sekali M. 1997. «L'acquisition des premières prépositions chez un enfant francophone ». In Faits de Langue - La préposition:

une catégorie accessoire ? Ophrys : 201-211.

MoUnIN, G. 1974[1993]. Dictionnaire de la linguistique. Paris : Presses

Universitaires de France.

MURPHY, G. 1996. « On Metaphoric Representation ». Cognition, 60 : 173-

204.

MURPHY, G. L., et D.L. Medin 1985. « The role of theories in conceptual cohérence ». Psychological Review, 92 : 289-316.

PACHERIE, E. 1993. «L'hypothèse de la structuration des connaissances par domaines et la question de l'architecture fonctionnelle de l'esprit ». Revue Internationale de Psychopathologie, 9: 63-89

PALMER, F.R. 1978. Descriptive and Comparative Linguistics : a Critical Introduction. Oxford : Faber \& Faber.

PUCKICA, J. 2008. « Sens et relations de sens dans les grammaires de construction ». Lexis [en ligne], 1.

PETIT, J.-L. 1999. « Le langage est-il dans le cerveau ». Intellectica, 1999/2, 29. PUTNAM, H. 1981. Reason, Truth and History. Cambridge, Cambridge University Press.

Rakova, M. 2002. «The Philosophy of Embodied Realism: A High Price to Pay? » Cognitive Linguistics, 13 : 215-244.

RASTIER, F. 1991. Sémantique et recherches cognitives. Paris : PUF. RASTIER, F. 2000. «De la sémantique cognitive à la sémantique diachronique : les valeurs et évolutions des classes lexicales», Texto [En ligne], URL : http://www.revue-texto.net/index.php?id=557. RONAT. M. 1986. La grammaire modulaire, Paris, Les éditions de minuit. ROSCH, E. 1973. « Natural Categories ». Cognitive Psychology 4 : 328-350. ROSCH, E. 1974. « Linguistic Relativity ». In A. Silverstein (Ed.), Human communication: Theoretical perspectives. New York : Halstead. ROSCH, E. 1975. « Cognitive Representations of Semantic Categories ». Journal of Experimental Psychology, General, 104 : 192-233. RosCH, E. 1977. « Human Categorization ». N. Warren (Ed.), Advances in cross-cultural psychology, Vol. 1. London: Academic Press. ROSCH, E. 1978. « Principles of categorization ». Cognition and Categorization. Sous la direction de E. Rosch et B.B. Lloyd. Hillsdale, 
Erlbaum : 27-48.

ROSCH, E. 1983. « Prototype Classification and Logical Classification: The two systems ». E. F. Scholnick (ed.), New trends in conceptual representation: Challenges to Piaget's theory? Hillsdale, NJ : Erlbaum. ROSCH, E., et Mervis, C. B. 1975. Family Resemblances: Studies in the internal structure of categories. Cognitive Psychology, 7 : 573-605. ROSCH, E., et B. Lloyd (eds.). 1978. Cognition and Categorization. Hillsdale, NJ : Erlbaum.

Rosch, E., Mervis, C. B., Gray, W., Johnson, D., \& Boyes-Braem, P. 1976.

Basic objects in natural categories. Cognitive Psychology, 8 : 382-439.

SPELKE, E. S. 1998. « Nativism, empiricism, and the origins of knowledge.

Infant Behavior and Development », 21 (2) : 181-200.

VARELA, F., E. Thompson et E. Rosch. 1993. The Embodied Mind.

Cambridge : MIT Press.

VICTORRI, B. 2000. «Théories linguistiques et cognition ». In Cognito, revue

romane de sciences cognitives, $16: 1-6$

ZLATEV, J. 2008. « The co-evolution of intersubjectivity and bodily mimesis ».

In The Shared Mind, Zlatev, Jordan, Timothy P. Racine, Chris Sinha and Esa Itkonen (eds.) : 215-

244.

\section{NOTES}

1. Et ce depuis Kleiber, 1990 , p. 9

2. Water sera létal ou vital selon que l'item est considéré devant une source non potable ou face à une gourde dans un désert. L'évidence d'un tel jugement, révélant un flou référentiel autour de ce qu'il est entendu par catégorie, prototype, ou typicalité, nous suggère l'impossibilité même de cette question - les principes de catégorisation naturelle ou perceptive ne peuvent pas être employés pour rendre compte des extensions catégorielles de la langue, en particulier si l'on considère le langage comme devant recourir à un lexique mental complexe et figé (ce qui n'est pas notre cas), car la spontanéité de la catégorisation serait évidemment biaisée.

3. "In a default context, tea, coffee or coke might be listed as a typical beverage, bur wine is more likely to be selected in the context of a dinner party". (Rosch, 1999, p. 66).

4. C'est pour cette raison que la sémantique du prototype est exclusivement descriptive, (et non explicative) : comme la théorie de la catégorisation des objets concrets, elle se fixe pour objectif la description des classes constituées.

5. Seuls quelques textes le rapportent explicitement. Il ne s'agit jamais de figures de la LC, mais de commentateurs avertis (Dubois, 1991 ; Corrigan, 1989).

6. «La productivité des concepts mis en circulation par Rosh se trouve obérée par un certains nombres d'implicites invérifiables » (Dubois, 1991, p. 12). 
7. En 1978, Rosch met en garde contre le caractère métaphysique du monde ( $a$ world without a knower »), mais en 1981, elle s'attache à décrire la catégorisation des objets de ce monde au moyen de ses corrélats d'attributs («natural objects»).

8. C'est la raison pour laquelle on parle parfois de théorie probabiliste, plutôt que de théorie du prototype.

9. L'appréhension simple désigne, chez l'Aquinate, les connaissances qui ne sont pas discursives. Sont «simples": l'appréhension des sens externes, de l'imagination et de l'intelligence qui se saisit d'un objet de façon intuitive : « Sed dicendum, quod magis universalia secundum simplicem apprehensionem sunt primo nota, nam primo in intellectu cadit ens, ut Avicenna dicitet prius in anima cadit animal quam homo (...) ». (In Garceau, 1968, p. 131).

10. Ce sont, par exemple, les scènes pertinentes pour le savoir humain (humanly relevant scenes) qui expliqueraient le sens causal des signes de type " proposition ».

11. L'approche distribuée du langage (distributed language group) considère que la langue est un système symbolique dépendant de postures communicatives qui détermineraient les interactions humaines (la langue n'existerait pas en elle-même). Cette approche reprend une part non négligeable des théories de la cognition distribuée : le savoir n'est plus confinée aux individus ; la mémoire et le savoir, dont les connaissances techniques et les outils, sont placées, c'est-à-dire distribuées, dans « l'environnement » au travers des communautés (sociales) en particulier.

12. La locution au jour d'aujourd'hui manifeste certainement un effort supplémentaire de précision pour cibler l'une ou l'autre des acceptions, voire le point de départ d'une autre agglutination lexico-grammaticale.

13. La catégorisation dynamique ('online') est en effet de faible recours quand il s'agit de modéliser l'histoire des sens et de figements.

14. Pourquoi certains membres ou exemplaires catégoriels sont-ils saillants et deviennent des prototypes? La question n'est jamais abordée. Lakoff, au détour d'une intuition plus tard fortement critiquée par Kleiber (1990, p. 42), suggère à propos de la méthode de classification en CNS que les entités, qui sont ou ne sont pas des membres d'une catégorie, sont souvent ressenties comme identifiables et classifiables rigidement selon une folk theory of categorization (Lakoff, 1987, p. 5). On pourra douter du caractère explicatif de cette remarque, mais l'on concédera sûrement que, transposée aux prototypes, elle rejoindrait la position de Rastier sur la modularité diachronique des prototypes : valoriser des « entités » les rend prototypiques, et cela s'avère être dépendant des époques et des contextes sociaux. La perception et/ou la mélioration de professeur relativement à la catégorie figure de l'autorité ou figure du savoir serait très probablement discontinue.

15. On pourra trouver surprenant que ces fluctuations de valorisation constituent le critère exclusif de cette étude. Il paraît évident que la centralité d'un exemplaire dépend de sa réception sociale - en cela, cette entreprise est on ne peut plus légitime. Cependant, la critique faite à la LC s'avère réversible : certes, il n'est pas de prototypie diachronique dans la littérature cognitive, mais le principe de variation ne le proscrit-il pas? N'est-il pas rapportable à une étude des couches successives des états de la langue? Que Berlin et Kay, puis Rosh considère le prototype comme une entité naturelle et achronique est en effet problématique. Mais si l'on sépare prototypie naturelle et prototypie linguistique à partir de différences méthodologiques tangibles, il nous paraît peu acceptable de souligner une faiblesse épistémologique dans laquelle la LC se garde bien de tomber, même si elle ne se pose pas la question.

16. Kleiber remarque très justement qu'il persiste une large confusion terminologique autour des notions de prototype, principe dû à la psychologie cognitive et à l'anthropologie, de stéréotype l'acception est alors sémantique et consiste en la continuation des travaux de Putnam - puis celle de ressemblance de famille (Kleiber, 1991, p. 9). Nous remarquons aussi que ce vocable Wittgensteinien et les principes qui lui sont associés, parfois équivalent, parfois fondent le 
modèle psychologique de la prototypie (et non linguistique, comme développé dans ce chapitre), ce de manière très indistincte.

17. Rosch parle de la «structure du monde perçu. » Un exemplaire jugé représentatif d'une catégorie est en conséquence une entité concrète, objective, d'un environnement indépendant du sujet. (Rosch, 1978, p. 28).

18. On pourrait nous reprocher ici de ne pas prendre en compte la tentative de Lakoff (1987) à individualiser les prototypes (relativement au sujet cognitif); en cela, il est vrai, les prototypes ne sont plus naturels mais mentaux (en ce qui concerne les artefacts encore plus clairement, car Lakoff reconnaît tout de même l'existence des objets concrets du monde, même s'ils sont déformés par les sense-data). En revanche, les traits des scripts, scènes ou espaces mentaux sont figés, idéalisés, et communément partagés ; dès lors, si le sujet peut se désengager, et se servir de ces représentations mentales seulement comme repères cognitifs, il n'en reste pas moins que leur caractère universel revêt des aspects d'ontologie.

\section{RÉSUMÉS}

Cet article a pour ambition de questionner les fondements des approches catégoriales souples, non essentialistes, desquels dépend l'architecture théorique de modèles linguistiques actuels et notamment, ici, de la Linguistique Cognitive américaine. Celle-ci, en effet, considère acquises et indiscutables les données de la psychologie cognitive - il en va de son engagement, dit « cognitif », à embrasser les résultats des disciplines connexes des sciences de la cognition. Après avoir réexposé les points essentiels de la théorie roschéenne - au travers, en particulier, des quelques articles séminaux - nous soulignons les apories auxquelles elle a dû rapidement faire face et auxquelles elle a, seulement en partie, su réagir, questionnons la souplesse du modèle et sa critique, partiale, du modèle aristotélicien, et proposons de clarifier la problématique catégoriale en scindant prototypes conceptuel et sémantique. Nous arguons enfin que c'est au mépris des critiques disciplinaires internes que la LC a importé des pratiques qui fragilisent un pan entier de son assise épistémologique.

This article aims at questioning the foundations of non-essentialist categorial approaches on which contemporary linguistic models heavily rely. Cognitive Linguistics, in particular, considers unquestionable the data provided by cognitive psychology - the " cognitive commitment ", as it is called, indeed urges to embrace the results of related empirical disciplines. After reexposing the roschian theory - out of a reading of its seminal articles - the author underlines the contradictions it was soon to be mired in, questions its partial understanding of the aristotelician model, and proposes to clarify the categorial issue by separating conceptual and semantic prototypicality. The author argues this is despite disciplinary controversy that CL imported results and practises which eventually shake its epistemological ground.

\section{INDEX}

Mots-clés : catégorie, concept, linguistique cognitive, prototype, sciences de la cognition Keywords : category, Cognitive linguistics, concept, prototype 


\section{AUTEUR}

\section{JEAN-BAPTISTE GUIGNARD}

Université Technologique de Compiègne 